\title{
CLINICAL OBSERVATIONS ON THE INTRA-OCULAR TENSION OF OPIUM HABITUÉS IN FORMOSA
}

BY

\author{
Kanji Kanda and Kazen So \\ TAKAO, TAIWAN, JAPAN
}

THE most accurate estimation of the intra-ocular tension is obtained by using a manometer, but for clinical purposes this is not so convenient as the tonometer on account of its complexity of application, so that the latter is preferable. There are many tonometers of various design and forms but the one most widely used is that of Schiötz.

The intra-ocular tension estimated with this instrument shows a wide range of the normal limits in different individuals. As a rule, it is higher in the forenoon than in the afternoon; it is raised in the dark and after considerable physical exertion. Thus, it not only varies in different persons according to various conditions, but there are also considerable daily differences even in the same person. Moreover, it varies widely in individuals even in the same conditions under which it is estimated. Therefore it is of no special significance to compare the estimated value of the intraocular tension of any person with the average normal limit of the intra-ocular tension in order to decide whether the former value is normal or not, unless the general conditions of the examinee or the conditions under which the estimation is done are explained.

The average normal limit of the intra-ocular tension of the Japanese people is given more or less differently by many authors. It is, according to Professor Dr. Shoji of Kyushu Imperial University, from $19.00 \mathrm{~mm}$. $\mathrm{Hg}$ to $29.50 \mathrm{~mm}$. $\mathrm{Hg}$, the average being $24.21 \mathrm{~mm} . \mathrm{Hg}$, which is equivalent to that of Europeans.

Now it is generally known that the intra-ocular tension is raised when the pupil is dilated, as for example after instillation of a few drops of atropine solution, and lowered when the pupil is contracted. In the opium habitués, the contracted pupil or miosis is supposed to be one of the most characteristic features of the addiction. This contraction of the pupil is not caused by the local or direct action of opium or morphine, but is one of the central or indirect actions of the drug that paralyses the inhibitory action of the nervous mechanism on the parasympathetic nerve that supplies the sphincter pupillae. In other words, morphine causes, in this case, excitation of the latter nerve, i.e., is antagonistic to the sympathetic nerve which supplies the dilatator pupillae. In Osler's "The Principles and Practice of Medicine," eighth edition, 1919 , there is a description of the morphia habit in which it is stated "that the pupils, except when under direct influence of the 
drug, are dilated, sometimes unequal." May this not be a mistake, since morphine or opium has no direct action upon the pupil ? If it be true that the contracted pupil is always accompanied by lowered intra-ocular tension and the dilated pupil by increased intra-ocular tension, as is generally believed, is it also lowered in the opium habitués whose pupils are mostly contracted? This was the first question we had to settle.

Recent work on the pathogenesis of glaucoma has done much to clarify the nature as well as the cause of the disease.' One fact is that most of the glaucoma patients are supposed to be in a vagotonic condition, and this fact has been proved by many authors to be the case. Various experiments have also been done on the inter-relation between the autonomic nervous system, especially the sympathetic, and the mechanism of the rise or fall of the intra-ocular tension. But these results are not always uniform. As for example, in the experiment of Yada, direct stimulus upon the cervical sympathetic nerve caused a fall in the intra-ocular tension, while in the experiment of Bistis, the stimulus of the same nerve caused, on the contrary, a rise. Now the so-called abstinent symptoms or phenomena of the habit seen in the opium habitués such as diarrhoea, nausea, vomiting, lumbago, recurring colic, distressing restlessness and so forth, which occur when the use of opium has been suddenly withdrawn, are looked upon as those of the parasympathetico-tonic condition, and thought to be of the same nature as the vagotonic. Professor Abé of Keio University, has succeeded in proving this to be the case by experiments on the monkey, which is readily adaptable to opium addiction. He has also shown that morphine in the habitués causes the excitation of the ends of the parasympathetic nerves when it is withdrawn from the tissues. He established from this fact a reliable theory of the treatment of the opium habit. The principle of this theory is that the so-called abstinent symptoms or the symptoms due to the excitation of the parasympathetic nerves, which occur when the drug has been withdrawn abruptly, can easily be relieved by giving other narcotics, instead of morphine or opium which paralyse the ends of the said nerves and are not habit-forming, such as scopolamine and atropine. Now, if it be probable that most glaucoma patients are in the vagotonic condition as above mentioned, and conversely those who are in this condition may be more liable to glaucoma than the ordinary person, then is the raised intra-ocular pressure in the opium habitués who are, as a rule, in the parasympathetico-tonic condition, the same as the vagotonic one in its nature, This was the second question we had to settle.

Thirdly, although many authors suggest an intimate parallel inter-relation between the blood pressure and the intra-ocular 
tension, yet there are a few authorities who do not recognize this. However, on the other hand, none can deny the parallel relation between the capillary blood pressure of the eyeball or of the surrounding orbital tissues, whether there may be systemic evidence of hyperpiesia or hypopiesia. Now, in the opium habitués, there should be hypopiesia, since they are mostly in parasympatheticotonic or vagotonic condition as above mentioned. And this is proved to be the case. If the parallel inter-relation between the intra-ocular tension and the general blood pressure can be accepted, is the intra-ocular tension of the opium habitués lowered, since they are in a hypopiesic condition?

Besides these questions, at first we intended to obtain the graphs of the values of the intra-ocular tension and the daily blood pressure of each opium habitué during the treatment. Is there any common type of the curve values of the intra-ocular tension? Is the curve raised or lowered by the withdrawal of opium or the treatment of the habit? Is the rise or fall of the curve in co-relation with the rise or fall of the blood pressure curve? In order to know these facts, we estimated the intra-ocular tension and the blood pressure of each habitué once daily during the whole period of the treatment, and obtained their curves respectively. These results, however, were not satisfactory, especially as graphic observations of the daily differences of the intra-ocular tension and the blood pressure and their inter-relation, and also the average period of the treatment for each habitué were made over too short a period to give reliable statistics. Therefore, in order to have sufficient results for these purposes, we must take graphs of them during as long a period as possible both before and after the treatment, or if necessary, make experiments with such a readily opium-addicted animal as the monkey.

Our estimation of the intra-ocular tension and the blood pressure of the opium habitués was done from February 16 to April 16, 1931. Those opium habitués were all Formosan Chinamen, who were brought under observation during isolation in hospital. Now the question of the control of the opium habitués among the natives of Formosa dates from the time when this island was, for the first time, brought under the rule of Japan as the result of the China-Japanese war in 1895. At the time of the opening of the Japanese Government in Formosa in 1895, it was one of the most important questions how to control the opium habitués among the natives, as there were at that time a great many habitués, so that the Government has adopted the so-called "gradually reducing policy" for them. For this purpose, the Government granted the confirmed habitués to be the specially licensed ones, and on the other hand strictly prohibited them to use as well as to have opium secretly, and threatened to punish 
any secret opium habitué or smuggler if detected. The Government established a Monopoly Bureau for manufacturing and supplying opium, and supplied it only to those specially licenced habitués through definite agencies. However, as long as opium is looked upon as a panacea as well as the source of ecstasy by most of the Formosan natives, whether they are habitués or not, as it is among the Chinese people, it is difficult to reduce the number of secret habitués. For, even nowadays, notwithstanding the lapse of more than 35 years since Formosa has been brought under the rule of Japan, there can still be detected many secret habitués as well as opium smugglers who risk inspection by the police and officers of the Customs House. In the year 1929, the Government General of Formosa, having been encouraged by Professor Abés (Keio University) great success in establishing a theory for the treatment of the morphia habit and also having been forced by the conventional resolution of the Opium Conference opened at Geneva in 1925, renewed her decision to treat opium habitués on a large and most systematic scale in order to meet her ever consistent colonization policy of "assimilating the Formosan natives into the Japanese," on the one hand, and on the other from the humane point of view, to rescue the opium habitués. And so the Government has revised the Opium Ordinance for Formosa in 1929, and has given new licences to those who had been secret habitués, and at the same time ordered them to receive treatment. 'These were confirmed to be habitués through thorough medical examination and ordered admission to the ward newlybuilt for this purpose in 11 local Government hospitals and one central hospital at Taihoku. These newly licensed opium habitués numbered 13,584 at the end of the year 1929. Besides these, there had been 26,942 formerly granted special habitué's licences, the number of which was 165,762 in 1900 , and 50,597 in 1897, showing, therefore, much reduction since the Government was opened here in 1895. They are isolated at the special ward called "Kôseika" which means "Rebirth Department" under careful watch as well as strict inspection, so that they have no means of getting opium. All expenses, except for private purposes, are paid by the authorities. The diet is sufficiently good and nourishing. The principles of the treatment are chiefly based upon Professor Abé's theory, but here the habitués receive morphine internally in various doses according to the daily doses of opium for each habitué before admission for treatment, and also according to the strength of various distressing abstinent symptoms. This administration of morphine is to be decreased in doses as soon as possible in the early period of the treatment, and is withdrawn entirely when the patients no more complain of any uncomfortable symptoms without it. For the purpose of relieving the so-called 
abstinent symptoms, extract of roth is given until these disappear entirely. Solutions of grape sugar and chloride of calcium are also administered intravenously in order to relieve the sensations of fatigue and coldness of the extremities and extreme restlessness.

These habitués on whom we estimated the intra-ocular tension and the blood pressure were 22 in number. They were mostly healthy persons other than being habitués. Their average age was 47 years 6 months, the oldest being 62 years and the youngest 33 years. They had all been old habitués of long duration, on an average for 8-9 successive years of addiction, the longest being 35 years. If the degree of their addiction to opium is represented by the individual amount of daily doses of opium, though this is not a scientific representation, the average is 0.92 mommé (a little more than 3 grammes, 0:276 mommé being equivalent to one gramme) for smoking and 0.2 mommé (about one gramme) for eating. According to Professor W. H. White, in India, the daily dose of opium varies from 2 to 80 grs. per day, 4 to 12 grs. being a common average, and probably about the same amount is usually consumed by habitués of Western race. With reference to this, our habitués are all strong smokers. Among these 22 habitués, 15 were smokers, 2 were eaters, 4 were both smokers and eaters and the remaining 1 had been a voluntary abstainer from opium before entering hospital. The average length of stay in hospital was 10.5 days, the longest being 18 days, and the shortest being only one week.

The intra-ocular tension of each habitué was estimated, once daily, in the same eye throughout the whole period of treatment, and this was done between 3.30 and 4 o'clock in the afternoon, with Shiötz's tonometer using a 5.5 grammes weight. Before applying the tonometer, the eye was anaesthetised with 5 per cent. solution of cocaine, and the value obtained here is the one read off at the first time in every estimation, since repeated application of the instrument makes the value too low. The daily values of the intra-ocular tension of each habitué were averaged at the end of the treatment by the number of the days of the treatment, and each of these average values were again averaged by the number of the habitués. Among the averaged daily values of each habitué, the highest was $31.30 \mathrm{~mm}$. $\mathrm{Hg}$ and the lowest was $9.77 \mathrm{~mm}$. Hg. The average value of the averaged daily values of each habitue is $22.59 \mathrm{~mm}$. $\mathrm{Hg}$, which is within the normal limit of the values of the Japanese people. The highest value among all the daily values of perpetual habitués is 37.50 $\mathrm{mm} . \mathrm{Hg}$, and the lowest is $8.0 \mathrm{~mm}$. Hg. These latter two values are taken from those of the daily ones of the two habitués, of whom one has the highest averaged daily value of $31.30 \mathrm{~mm} . \mathrm{Hg}$, and the other has the lowest averaged daily value of $9.77 \mathrm{~mm}$. $\mathrm{Hg}$. 
These two habitués did not show any noticeable affections of the eyes, except trachoma in the latter. Among all the habitués brought under estimation, nine had trachoma.

The majority have contracted pupils the size of which was observed in the dark room in about five minutes after entering the room. The average diameter of them was $2.8 \mathrm{~mm}$., so that this should be still less when observed in the daylight of an ordinary room.

Although the average intra-ocular tension of the opium habitués is nearly normal, since it is $22.59 \mathrm{~mm}$. $\mathrm{Hg}$, and this is within the normal limit of the intra-ocular tension, yet there can be seen a wide range of individual differences. Moreover, even in the same person, the intra-ocular tension differs more or less daily. Therefore, it was quite impossible to demonstrate common features in the curve values of the intra-ocular tension of opium habitués. In other words, the curves of each habitué are, if they are traced, irregular in themselves, and do not seem to be much influenced by the withdrawal of opium or the treatment of the habit. But only in a few cases, can there be traced in the curves a common tendency to rise after the administration of morphine has been ceased. This tendency to rise, however, is so slight that it may escape observation. This slight rise of the curves soon comes down as it approaches the ends of the curves.

The reason why the curves were irregular, and there were no common features about them, may be explained by the fact that the general condition of the opium habitués is quite unstable; since they are both mentally and physically affected. Therefore, we think the irregular form of the curves of the intra-ocular tension of the opium habitués is, in itself, one of the characteristic features of the habit.

We did not find either definite or parallel inter-relations between the intra-ocular tension and the blood pressure of the opium habitués. The arterial blood pressure of the arms of the habitués on the same side as that of the eye in which the intra-ocular pressure was taken, was estimated every day with Tycos tonometer just before the ocular estimation was done. We found rather opposite inter-relation between them, for the blood pressure was, in general, either somewhat lowered(hypopiesia) or at least normal, while the values of the intra-ocular tension were not correspondingly lowered, but remained normal or even a little higher than this. The systolic blood pressure was $113 \mathrm{Hg}$ and the diastolic $75 \mathrm{~mm}$. $\mathrm{Hg}$ on an average. 'These average values of the blood pressure are obtained in the same way as in obtaining the averaged values of the intra-ocular tension already mentioned.

From these observations, the authors finally came to the following conclusions. 
1. Although the opium habitués have mostly contracted pupils, and in the authors' cases their average size was $2.8 \mathrm{~mm}$. in diameter, the intra-ocular tension is either normal or somewhat higher than the normal, the average values being $22.59 \mathrm{~mm}$. $\mathrm{Hg}$ with Schiötz's tonometer using the 5.5 grammes weight.

2. Although the opium habitués are in a parasympathetico-tonic or vagotonic condition, and there is the fact that most of the glaucoma patients are also said to be in this condition, and conversely those who are in this condition may be more susceptible to glaucoma, the intra-ocular tension of the opium habitués is, in general, normal, though in a few habitués it was either a little higher or even lower than the normal limit of the intra-ocular tension.

3 . The curves of the values of the intra-ocular tension of the opium habitués are quite irregular in themselves, and have no feature in common. This fact may be looked upon as characteristic of the addiction. These curves do not seem to be much influenced by the withdrawal of opium or the treatment of the habit.

4. The blood pressure of the opium habitués is, on the whole, hypotonic, the systolic pressure being $113 \mathrm{~mm}$. $\mathrm{Hg}$ and the diastolic $75 \mathrm{~mm}$. $\mathrm{Hg}$ on an average. However, the intra-ocular tension is normal or even a little higher than the normal, so that there can be found no definite parallel relation between them.

Special thanks are due to Professor Dr. Kubo, master of the Government Takao Hospital, by whose kind permission these observations have been completed. (October 20, 1932.)

\section{REFERENCES}

1. White, W. H.-Textbook of pharmacology and therapeutics. 1901.

2. Osler.-The principles and practice of medicine. Eighth edition.

3. Government-General of Formosa.-Opium system and control of narcotic drugs in Formosa, Japan. February, 1930.

4. Yada, S.-On the influence of the sympathetic nerves upon the intra-ocular tension. Ganka-rinshio-iho. No. 304.

5. Bistis.-Le rôle du sympathetique dans le glaucome. (Abstract). Arch. d'Ophtal., February, 1930.

6. Shoji, Y., and Suzuki, S.-Vergleichende Studien ueber Ophthalmometer. Acta Societatis Ophthalmologicae Japaonicae. Tomus 35. Fasciculus 5. 1931. 